\title{
La corrupción como problema público en la televisión argentina: interacción entre medios y audiencias*
}

\author{
Esteban Zunino** \\ Brenda Focás**
}

Recibido: 2020-09-15 • Enviado a pares: 2020-09-20

Aprobado por pares: 2020-11-16 • Aceptado: 2021-01-19

https://doi.org/10.22395/angr.v20n39a6

\begin{abstract}
Resumen
La corrupción irrumpió como un problema público en la Argentina durante la década de los noventa, promovido por un conjunto de grupos sociales emergentes que lo instituyeron a partir de estrategias de denuncia. Ya en los años dos mil, el asunto se estabilizó en las agendas mediática y pública, aunque ligada a otros actores, casos y discursos circulantes. El presente trabajo pretende analizar la relación entre la construcción mediática la corrupción y su percepción pública en los cuatro conglomerados urbanos más importantes de Argentina: Área Metropolitana de Buenos Aires (AMBA), Córdoba, Rosario y Mendoza. A partir de un abordaje metodológico que triangula grupos focales desarrollados en las cuatro ciudades y un análisis de contenido sobre las noticias en los noticieros centrales de los canales trece y once de Buenos Aires, diez y ocho de Córdoba, tres y cinco de Rosario y nueve y siete de Mendoza, se pretende analizar cuál es la relevancia mediática y social del problema. Los resultados evidencian una alta importancia del tema en las agendas informativas, sobre todo en las de los medios de Buenos Aires, que presentan una cobertura partisana del problema. En relación con los públicos, el trabajo muestra que, si bien las audiencias expresan cierta distancia y desinterés con estas noticias, se observan procesos de recepción que pululan entre la polarización afectiva y la saturación cognitiva frente al tema.
\end{abstract}

Palabras clave: corrupción, televisión, audiencias, medios de comunicación.

\footnotetext{
El presente trabajo forma parte de los resultados del Proyecto de Investigación Orientado Conicet-Defensoría del Público "De la propiedad a la recepción. Estudio integral del circuito productivo de las noticias sobre delito e inseguridad en los noticieros televisivos de mayor audiencia de la Argentina (PICT 2015-2019)".

." Doctor en Ciencias Sociales y Humanas, Universidad Nacional de Quilmes, Argentina. Investigador del Consejo Nacional de Investigaciones Científicas y Técnicas (Conicet), Argentina. Profesor e investigador de la Universidad Nacional de Cuyo, Argentina. Correo electrónico ezunino@mendoza-conicet.gov.ar. Orcid: https://orcid.org/0000-0002-2951-9872.

... Doctora en Ciencias Sociales, Universidad de Buenos Aires, Argentina. Investigadora del Consejo Nacional de Investigaciones Científicas y Técnicas (Conicet), Argentina. Profesora e investigadora de la Universidad Nacional de San Martín, Argentina. Correo electrónico bfocas@conicet.gov.ar. Orcid: https://orcid.org/0000-0002-1749-6757.
} 


\title{
Corruption as a social problem in Argentine TV: interaction between media and audiences
}

\begin{abstract}
Corruption emerged as a public problem in Argentina during the 1990, promoted by emerging social groups that instituted it based on reporting strategies. Already in the 2000s, the issue stabilized in the media and public agendas, although linked to other actors, cases, and discourses. This paper aims to analyze the relationship between the media construction of corruption and its public perception in the Argentinian major cities: Buenos Aires, Cordoba, Rosario, and Mendoza. Based on a methodological approach that triangulates focus groups and a content analysis of the central news programs of channels 13 and 11 of Buenos Aires, 10 and 8 of Cordoba, 3 and 5 of Rosario and 9 and 7 of Mendoza, it is intended to analyze the mediatic and social relevance of the problem. Results evidence a high relevance of corruption in the news agendas, especially in those of the Buenos Aires media, which is consistent with some audience's perceptions. A partisan coverage awakens a polarized and affective reception that affects the processes of victimization. Likewise, the greater visibility of the issue in Buenos Aires raises divergences linked to the geographical variable, both at the level of content and perception. In relation to the public, results show that, although the audiences express a certain distance and disinterest, reception processes are observed that swarm between affective polarization and cognitive saturation.
\end{abstract}

Keywords: corruption, television, cultural user, mass media, communication, information, politics, Argentina.

\section{Corrupção como problema da TV argentina: interação entre mídia e público}

\begin{abstract}
Resumo
A corrupção surgiu como um problema público na Argentina durante os anos 1990, promovido por um grupo de grupos sociais emergentes que a instituíram com base em estratégias de denúncia. Já na década de 2000, o tema se estabilizou na mídia e nas agendas públicas, embora vinculado a outros atores, casos e discursos circulantes. Este artigo tem como objetivo analisar a relação entre a construção midiática da corrupção e sua percepção pública nos quatro conglomerados urbanos mais importantes da Argentina: Buenos Aires, Córdoba, Rosário e Mendoza. Com base em uma abordagem metodológica que triangula grupos focais desenvolvidos nas quatro cidades e uma análise de conteúdo das notícias nos telejornais centrais dos canais 13 e 11 de Buenos Aires, 10 e 8 de Córdoba, 3 e 5 de Rosário e 9 e 7 de Mendoza, pretende-se analisar a relevância midiática e social do problema. Os resultados mostram uma alta relevância do assunto nas pautas noticiosas, especialmente na mídia de Buenos Aires, o que é consistente com algumas percepções dos públicos. A cobertura partidária do tema desperta uma recepção polarizada e afetiva que afeta os processos de vitimização. Da mesma forma, a maior visibilidade do tema na mídia de Buenos Aires suscita divergências em função da variável geográfica, tanto no nível do conteúdo quanto na percepção da relevância do problema. Em relação ao público, o trabalho mostra que, embora os públicos expressem certo distanciamento e desinteresse por essas notícias, observam-se processos de recepção que pululam entre a polarização afetiva e a saturação cognitiva com o sujeito.

Palavras-chave: corrupção, televisão, usuário cultural, meios de comunicação de massa, comunicação, informação, política, Argentina.
\end{abstract}




\section{Introducción}

La corrupción irrumpió como un problema público en Argentina durante la década de los años noventa (Pereyra, 2009) impulsado por un conjunto de colectivos sociales emergentes que lo instituyeron a partir de estrategias concretas de denuncia. Desde ese momento, y asumiendo formas discursivas y estéticas similares, el asunto ganó visibilidad en las agendas mediática, pública y política. Ya en los años dos mil, la corrupción retornó con fuerza al debate, aunque ligada a otros actores, casos y discursos circulantes.

Si bien en su construcción social convergen diferentes tipos de delitos, por la naturaleza del problema, figuras implicadas, tratamiento periodístico y percepción pública, el problema no suele ser asociado ni por los medios, ni por las audiencias, a la noción de "inseguridad". En relación con la recepción, estas noticias son enmarcadas, en general, en la sección "política" y su incidencia en la vida cotidiana es experimentada con distancia. En este sentido, las noticias de corrupción promueven un interés relativo en las audiencias, ligado a los contextos específicos en que los casos se desarrollan, aunque despiertan ciertas emociones y reacciones vinculadas con las ideologías previas de quienes las consumen (Aruguete y Calvo, 2020).

No obstante, los casos más resonantes, generalmente vinculados con figuras importantes del ámbito público y privado, ocupan buena parte de las agendas de los noticieros más consumidos del país y forman parte de un abanico de temas que se presentan como amenazas concretas para la vida cotidiana. En términos de Thompson (2012), los casos de corrupción son presentados como escándalos políticos y promueven mecanismos de identificación afectiva que, a partir de una evaluación moral negativa sobre la figura del denunciado, llevan a la acción mediante el reconocimiento de una afectación directa que es sucedida con la indignación como respuesta (Pereyra, 2009).

En ese contexto, y producto de la creciente importancia de la corrupción en las agendas, tematizada como una forma novedosa de definir el acontecer político que tiene repercusiones concretas sobre las percepciones sociales y los sistemas de gobierno, el presente trabajo pretende analizar el proceso de instalación de la corrupción como problema público en los noticieros de televisión más importantes de Argentina y, a partir de ello, hipotetizar sobre la relación entre los contenidos mediáticos y su recepción.

\section{Antecedentes y perspectivas teóricas}

Si bien estudios como el del Centro de Economía y Finanzas para el Desarrollo de la Argentina (Cefid - Ar) calculó que para las primeras dos décadas del siglo XXI solo 
el $5 \%$ de los fondos fugados que afectan directamente al erario público nacional proceden de hechos de corrupción gubernamental; el $31 \%$, de actividades criminales (narcotráfico, venta de armas, entre otros) y el $64 \%$, de maniobras destinadas a evadir el pago de impuestos, con los Panamá Papers y los Paradise Papers a la cabeza, la corrupción es susceptible de ser pensada como un problema público central que afecta directamente la vida de los ciudadanos. Es decir, como un asunto que, en el marco de la competencia temática con otros, logra adquirir consenso social sobre su importancia, movilizar la expresión ciudadana y de especialistas sobre su relevancia, apelar a diferentes esferas del Estado para su resolución y, finalmente, estabilizarse como preocupación social (Kessler, 2014). Por lo tanto, el aumento —o no- de actos de corrupción en el ámbito público no explica por sí mismo la creciente preocupación social por este asunto.

Diferentes investigaciones dan cuenta de que logró instalarse como un tema central de la opinión pública a partir de la denuncia de escándalos políticos durante la década de los noventa (Palau y Davesa, 2013; Pereyra, 2009). Asociada en Argentina a diferentes acontecimientos que se sucedieron durante las presidencias de Carlos Menem (1989-1999), adquirió una estética propia que inició una serie en la que se inscribieron nuevos casos.

La Sociología de los Asuntos Públicos constituye un abordaje operativo para su análisis, rico en antecedentes. Inscrita críticamente en una corriente constructivista de los problemas sociales (Kitsuse y Spector, 2001) que pone en tela de juicio la naturaleza objetiva atribuida a los hechos por la sociología funcionalista, forma parte de la perspectiva de la construcción social de la realidad (Berger y Luckmann, 1968). Según esta corriente de indagación teórica, la principal condición para que un problema social pueda instalarse socialmente es que sea instituido a partir de una demanda. Cuando estas se vuelven públicas y tienen una repercusión exitosa son capaces de generar procesos de estabilización discursiva que las tornan reconocibles y que actúan como series ante la irrupción de nuevos asuntos del mismo tipo (Kessler, 2014; Pereyra, 2012), además de obligar al poder político estatal a pronunciarse. En tanto su proceso de estabilización es determinante en la elaboración de representaciones colectivas o esquemas de interpretación que orientan la experiencia práctica.

Concebir a la corrupción de este modo implica reconocer algunos componentes típicos que operan como condiciones de posibilidad para su instalación en el debate ciudadano. El primero de ellos es la publicidad. Concebida como "los mecanismos mediante los cuales un tema se vuelve público" (Pereyra, 2009, p. 6), constituye una dimensión sobre la cual los medios de comunicación ejercen un rol central, en la medida en que funcionan como maquinarias propaladoras de los sucesos, aunque también como denunciantes. 
Si bien la corrupción suele presentarse como un escándalo político (Thompson, 2012), se diferencia de otros por una serie de especificidades que la identifican como un asunto particular. En términos de Pereyra (2012), la transgresión constituye un primer elemento distintivo de la corrupción como problema público, en tanto implica la ruptura necesaria de una o varias normas sociales. Fundamentalmente, la transgresión implica una evaluación sobre esa ruptura que diferencia moralmente al denunciante - individual o colectivo - del denunciado. En la medida en que la transgresión sea dada a publicidad, otro requisito indispensable para que se constituya en problema social, el público ingresa a la discusión como actor participante, en tanto se vea interpelado afectivamente en la situación.

El tercer componente distintivo de la corrupción es la controversia. Generada en un comienzo a partir de la definición de las identidades del denunciante y el denunciado, la estética sensacionalista de la mediatización de la corrupción como escándalo político moviliza aliados y detractores que se estabilizan a partir de expresiones públicas de repudio. La controversia puede ser definida a partir de dos componentes. El primero de ellos es la degradación presente como discurso moralizador oprobioso que reprocha y reprende. Este tipo de discurso, que realza los rasgos morales positivos del denunciante, se sustenta en la instalación de una prueba como elemento central que aporta verosimilitud y plausibilidad a la denuncia. En ese sentido, resulta pertinente poner en claro que "Ip|robar, no implica principalmente demostrar la adecuación entre la denuncia y la realidad de ciertos hechos sino aportar elementos que permitan formar un juicio subjetivo sobre los hechos en cuestión" (Pereyra, 2012, p. 10).

Ahora bien, ¿̇cuál es el rol de los medios de comunicación en la generación e instalación de escándalos de corrupción? Algunas perspectivas teóricas a partir de las cuales se han desarrollado investigaciones empíricas sobre el tema aportan elementos conceptuales relevantes a la hora de abordar el interrogante.

En primer lugar, la teoría de la Agenda Setting, que sostiene que los medios de comunicación son actores centrales en la instalación de temas y aspectos en la opinión pública, indaga sobre las condiciones contingentes que realzan o atenúan los efectos mediáticos cognitivos. En relación con los temas de corrupción, una distinción fundamental a tener en cuenta es la diferenciación entre temas experienciales (obstrussive issues), los cuales producto de la cercanía con la experiencia directa intersubjetiva atenuarían la centralidad de las representaciones mediáticas; y aquellos no experienciales, aquellos que debido a su menor posibilidad de contrastación directa a través de la experiencia serían más permeables al moldeado mediático (McCombs y Valenzuela, 2014; Zucker, 1978).

En función de la operacionalización propuesta se puede pensar a la corrupción como un tema no experiencial sobre el cual los medios de comunicación adquieren 
centralidad en su definición. Ya sea como dispositivos indispensables para la publicidad de los casos (Pereyra, 2014) o como actores denunciantes (Palau y Davesa, 2013), constituyen un eslabón fundamental en la cadena de montaje de los escándalos políticos. Esos escándalos, en tanto, se estabilizan y renuevan a partir de la presentación mediática de series de acontecimientos que los torna reconocibles y reactualiza como tema de discusión. En ese sentido, del mismo modo que los casos de inseguridad presentados en términos de olas que se construyen a partir de "casos conmocionantes" (Fernández Pedemonte, 2010), los escándalos de corrupción también modifican los criterios de selección de los periodistas, lo que incrementa su relevancia y visibilidad. En términos teóricos, la relevancia de un tema puede operacionalizarse en dos dimensiones: la frecuencia, repetición de piezas informativas sobre un asunto, y la jerarquía que adquieren esas piezas dentro de las agendas mediáticas (McCombs, 2006) factores que resultan determinantes para la importancia percibida por parte de las audiencias.

De este modo, tal como entiende Pereyra (2012) "la actividad periodística está directa o indirectamente entrecruzada con la producción de escándalos y ese proceso de producción involucra el desarrollo de una estética del escándalo que les otorga ciertos rasgos propios, peculiares y una temporalidad específica" (p. 6), elementos que contribuyen con su instalación como preocupaciones públicas relevantes.

En el mismo sentido, resulta posible considerar que las representaciones de la corrupción son portadoras de encuadres que están en tensión y pugnan por la definición social del problema. Encuadrar una situación supone "seleccionar algunos aspectos de la realidad percibida y hacerlos más relevantes en un texto comunicativo, de modo que se promueva una determinada definición del problema, una interpretación causal, una evaluación moral y/o una recomendación de tratamiento para el asunto descrito" (Entman, 1993). Si se tiene en cuenta que los juicios subjetivos son centrales en la definición social de los problemas públicos, y que por dicha definición, atribución causal y evaluación moral pugnan los diferentes actores involucrados, resulta posible considerar la centralidad que adquieren los medios en la construcción de los escándalos de corrupción y en la elaboración de esquemas estables, persistentes y transmisibles para su reconocimiento (Reese, 2001).

En la transmisión de dichos encuadres juegan un rol fundamental las fuentes de información, caracterizadas como "personas o grupos de personas, organizadas o no, que conocen los hechos que el periodista acabará por convertir en noticia" (García Santamaría, 2010, p. 521), suelen mantener con los periodistas relaciones transaccionales y simbióticas que las hacen partícipes del proceso de elaboración de la información (Bagdikian, 1985). 
Por último, un elemento resulta vital para el análisis de los procesos de mediatización del asunto que aquí se investiga. Las coberturas de la corrupción suelen recaer sobre las características personales de sus protagonistas, cuyas posiciones se construyen de manera fuertemente moral a partir del binomio denunciante-denunciado. Este tipo de tratamiento informativo da cuenta de un sesgo típico de las coberturas mediáticas sensacionalistas: la personalización de la noticia, la cual expresa la tendencia mediática a focalizar su atención en los actores más que en sus acciones, debido a la naturaleza dramática del interés humano en las historias (Bennett, 1991). Tal decisión editorial, que tiene por fin incrementar la atención del público a partir del despliegue de elementos afectivos explícitos dramáticos y controversiales (Martini, 2017; Palau y Davesa, 2013), contribuyen con un enfoque episódico (Iyengar, 2001) que tiende a descartar los componentes estructurales del problema en pro de una cobertura descontextualizada y fragmentada. En contextos convergentes, la dramatización mediatizada de los escándalos políticos, entre otros temas que componen las agendas, puede contribuir a fomentar escenarios polarizados de exposición selectiva (Aruguete y Calvo, 2018; Waisbord, 2020) y, consecuentemente, desinformación (Bennett y Livingston, 2018; Bennett y Pfetsch, 2018).

\section{Metodología}

El presente trabajo tiene como fin analizar la construcción mediática de la corrupción como problema público en Argentina y contrastar los contenidos informativos de los principales noticieros del país con las percepciones de las audiencias. En términos específicos, se procura establecer qué tipo de tópicos y temas condensan en el concepto de corrupción en los contenidos informativos de la televisión, quiénes son las fuentes de información sobre los diferentes asuntos relacionados con el problema y qué tipo de percepciones generaron las noticias de corrupción en las audiencias.

En función de los objetivos, se plantean las siguientes preguntas de investigación:

Pl: ¿Cuál fue la relevancia de la corrupción en los principales noticieros de televisión de Argentina?

P2: ¿Qué tipo de actores se convirtieron en fuentes de información en el tratamiento noticioso del caso?

P3: ¿Cuál fue la dinámica de las percepciones de las audiencias de los principales noticieros de televisión de Argentina sobre corrupción?

A partir de los objetivos planteados se desarrolló una estrategia metodológica consistente en la triangulación entre el análisis de contenido cuantitativo y el desarrollo de grupos focales para el estudio de los procesos de recepción. El concepto de trian- 
gulación deriva de la geometría aplicada a la navegación y es utilizado metafóricamente para definir un objeto de estudio a partir del entrecruzamiento de líneas trazadas desde distintos puntos de vista. El contraste de las diferentes perspectivas ofrece una imagen más completa de un objeto, para lo cual es necesario definir la ubicación desde la cual se orienta cada teoría y reducir su sesgo (Gallart, 2002). Se trata de una aproximación que aporta conocimiento interpretativo y permite elaborar hipótesis complementarias de aquellas que enuncian regularidades. El abordaje planteado para este trabajo tiene la particularidad de orientar su atención a unidades comunes de observación sobre dos instancias diferentes del circuito informativo: los contenidos de las noticias y su consumo.

El análisis de contenido permite examinar los mensajes mediáticos, reconstruir su arquitectura, conocer su estructura, sus componentes básicos y su funcionamiento; y realizar inferencias reproducibles y válidas sobre fenómenos distintos a los directamente observados (Neuendorf, 2002). Mediante mecanismos lógicos, el analista saca deducciones sobre la porción de la realidad a la que alude la información, así como el contexto en que esta es producida (Colle, 2011; Igartua y Humanes, 2004; Krippendorff, 1990).

En función de los objetivos planteados, se analizaron todas las noticias sobre corrupción de una semana construida durante el mes de agosto de 2016 de las ediciones centrales (nocturnas) de los noticieros de los canales once y trece de Buenos Aires, tres y cinco de Rosario, ocho y diez de Córdoba y siete y nueve de Mendoza.

El recorte temporal es intencional y no tiene pretensiones de representatividad del año completo. Se justifica debido a que, una vez iniciado el gobierno del expresidente Mauricio Macri (2015-2019), la corrupción se convirtió en un asunto que tuvo un alto nivel de relevancia en las agendas mediática y política. El inicio de trámite judicial contra exfuncionarios del gobierno anterior que involucraron directa o indirectamente a la expresidenta Cristina Fernández de Kirchner (2007-2015) se encontraba para el mes de agosto estabilizado, habiéndose producido las primeras imputaciones por parte del Poder Judicial. En ese sentido, la decisión fue tomada teniendo en cuenta que en Argentina el mes de agosto es el primer mes de alta actividad institucional del segundo semestre, luego del receso invernal de julio, y está alejado de la campaña electoral previa. En ese sentido, si bien los resultados de este trabajo no son generalizables al año 2016, permiten establecer ciertas tendencias sobre al problema que se investiga. Pero, sobre todo, establecer cruces entre contenidos informativos y recepción sobre un problema público de fuerte impacto social.

La selección de los canales de televisión se sustenta en que son los de mayor audiencia de los cuatro conglomerados urbanos más poblados del país. De este modo, 
se construyó un corpus de ciento sesenta y dos noticias que constituyen las unidades de análisis de esta investigación.

Para el análisis de contenido se tuvieron en cuenta las siguientes variables, en función de los objetivos planteados':

Tipo de delito: sistematiza una tipología para integrar diversos acontecimientos a partir de su similaridad semántica. Incluye las siguientes categorías:

1. Robo y hurto

2. Secuestro

3. Asesinato

4. Narcotráfico

5. Amenaza

6. Violencia sexual

7. Feminicidio

8. Violencia de género

9. Violencia intrafamiliar

10. Violencia infantil

11. Violencia Institucional

12. Violencia en cárceles

13. Violencia en las calles o en la comunidad

14. Violencia laboral

15. Corrupción en el ámbito público

16. Corrupción en el ámbito privado

17. Delitos medioambientales

18. Seguridad vial

19. Salubridad

20. Inseguridad Informática

21. Otros.

' Este trabajo forma parte de un proyecto mayor orientado al análisis del circuito informativo de noticias sobre diversos tipos de ilícitos. Para un conocimiento mayor de la matriz de análisis completa revisar Aruguete et al. (2018). 


\section{Dimensión geográfica:}

1. Área Metropolitana de Buenos Aires

2. Rosario

3. Córdoba

4. Mendoza

Victimario: se listan los nombres de las personas o instituciones sindicadas como responsables del problema.

Fuente: en función de la definición de Steele (1997) se considera

1. Fuente oficial: aquellas que provienen del ámbito estatal

2. Fuente no oficial: aquellas personas o instituciones que no provienen del ámbito estatal.

Seguidamente, el estudio de la recepción de noticias se desarrolló desde un enfoque cualitativo orientado por la herramienta del grupo focal, tendiente a comprender los sentidos atribuidos a las noticias sobre corrupción. Esta metodología permite observar las interacciones grupales donde se negocian sentidos de lo recibido (Arboleda, 2008; Bloor et al., 2000) en este caso a partir del consumo mediático personal. El fin fue caracterizar la interacción entre variables que gravitan en el interés o preocupación por la corrupción, las percepciones sociales; así como el acceso, la apropiación de la información y el impacto de las noticias en las escalas local-nacional.

Con este fin se conformaron, en cada región, grupos de diez participantes divididos por cuotas en función de las variables etaria, geográfica, socioeducativa y de género, con el fin de poder encontrar recurrencias y diferencias entre grupos y/o regiones: Ciudad de Buenos Aires y área metropolitana del conurbano, Rosario, Córdoba y Mendoza.

Para el trabajo en los grupos se diseñó una guía de pautas para poner a prueba distintas hipótesis y hallazgos internacionales sobre recepción de noticias. Específicamente, los doce grupos fueron segmentados de la siguiente manera: En Ciudad de Buenos y área metropolitana se realizaron seis grupos de diez personas cada uno. 1: dieciocho-treinta años. NSE C1-C2. Residentes en CABA, todos los barrios, menos Sur. 2: cuarenta-sesenta y cinco años. NSE C1 y C2. Residentes en CABA, todos los barrios, menos Sur. 3: veinte-cincuenta años. NSE D1, D2. Residentes en barrios Sur de la CABA (Lugano, Soldati, Barracas, Boca, Constitución, Floresta si bien no es Sur, Balvanera). 4: treinta-cincuenta años. NSE C1 -C2. Residentes en GBA primer cordón, Norte, Sur y Oeste. 5: dieciocho-treinta años. NSE D1- D2. Residentes 
en GBA segundo y tercer cordón. 6: cuarenta-cincuenta y seis años. NSE D1 D2. Residentes en GBA segundo y tercer cordón. En las ciudades de Córdoba, Rosario y Mendoza se trabajó con dos grupos en cada ciudad Grupo 1: veinticinco a cincuenta y cinco años. NSE bajo, y el otro grupo de veinticinco a cincuenta y cinco años NSE medio-alto. Los participantes fueron en partes iguales varones y mujeres y fueron reclutados por personal especializado mediante método bola de nieve, asegurando que no se conocieran entre ellos y que hubiera en cada grupo diversidad etaria, de inserción laboral y que provinieran de distintas zonas o barrios de cada ciudad. Los participantes recibieron una gratificación económica financiada por el proyecto, lo que aseguró la asistencia e interés de los participantes. Todos los encuentros se llevaron a cabo entre los meses de marzo y agosto de 2017. Para la discusión en los grupos focales se utilizaron como disparadoras noticias recolectadas durante agosto de 2016 que forman parte del corpus sometido a análisis de contenido².

\section{Resultados}

\section{La corrupción en los medios}

El análisis de los contenidos informativos de los principales noticieros de televisión de Argentina desarrollado por el proyecto Pio Conicet-Defensoría del Público "[d]e la propiedad a la recepción. Estudio integral del circuito productivo de las noticias sobre delito e inseguridad en los noticieros televisivos de mayor audiencia de la Argentina (PICT 2015-2019)" (Araguete et al., 2018), da cuenta de que, si bien la corrupción posee modos de construcción discursiva que la diferencian de los delitos típicos relacionados con la "inseguridad", a saber, ilícitos concretados o no contra la propiedad y/o las personas (Kessler, 2009), esta concentra fuertemente la atención mediática al punto de convertirse en el tipo de ilícito más relevante en los noticieros. En efecto, el 38,8 \% de las noticias referidas a delitos recayó en casos resonantes tematizados como escándalos de corrupción.

Además de una alta frecuencia de cobertura, los casos de corrupción adquirieron un importante nivel de jerarquía, la cual es posible determinar a partir de su habitual presencia en los titulares y anticipos, partes más visibles del noticiero en la que se presentan los temas del día. En efecto, cinco de cada diez noticias sobre corrupción integraron esos espacios que resultan centrales para identificar cuáles son los temas que los medios de comunicación pretenden instalar como importantes para el debate público.

\footnotetext{
${ }^{2}$ Las fechas de recolección de corpus y realización de los grupos focales estuvieron condicionadas por la disponibilidad de fondos y avance de la investigación en el proyecto Pio Conicet-Defensoría del Público "de la propiedad a la recepción. Estudio integral del circuito productivo de las noticias sobre delito e inseguridad en los noticieros televisivos de mayor audiencia de la Argentina (PICT 2015-2019)".
} 
Ahora bien, dos variables resultan importantes para comprender la particularidad del tratamiento informativo de este fenómeno. Tal como lo evidencia el gráfico 1, mientras que la cobertura mediática de la corrupción en el ámbito público acaparó el 34,8\% del hueco informativo total destinado a todos los tipos de ilícitos posibles, constituyendo el problema social relacionado con una infracción a una norma más visible en los noticieros, la corrupción en el ámbito privado apenas alcanzó el 3,8 \% de la cobertura informativa.

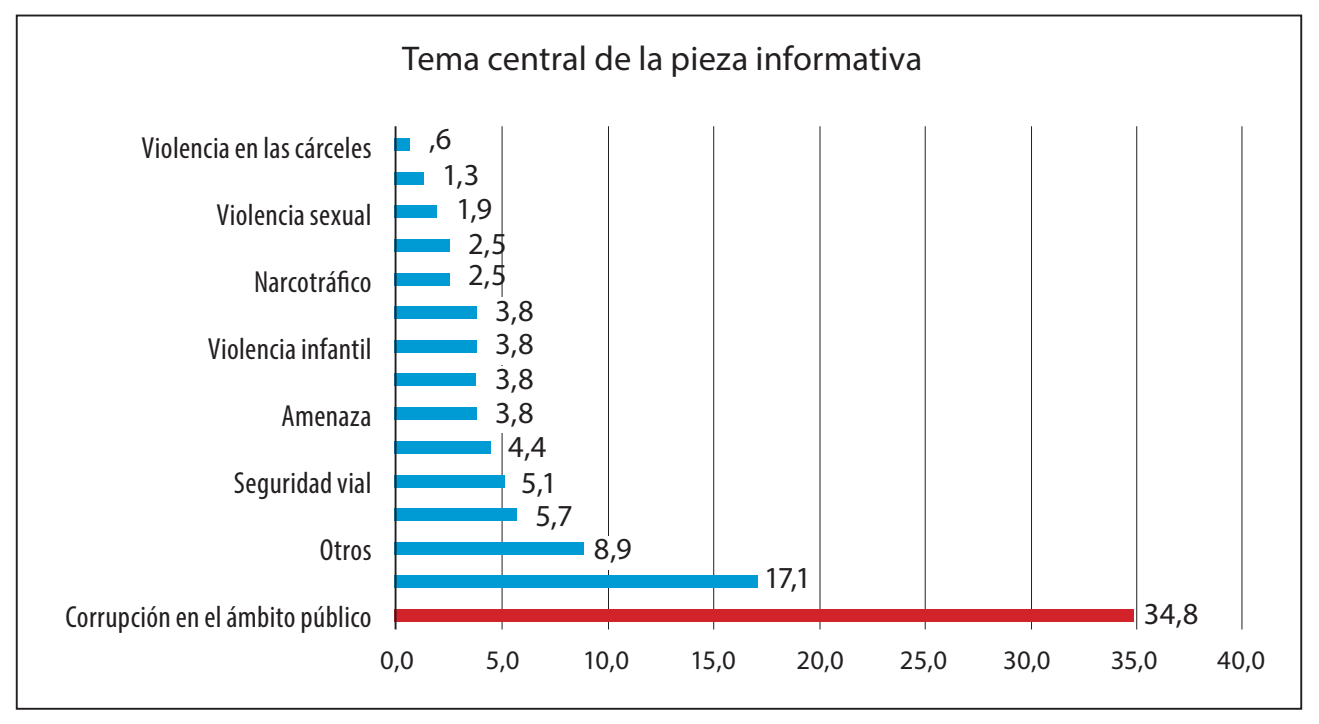

Gráfico 1. frecuencia de temas en la agenda mediática. Canales once y trece de Buenos Aires; tres y cinco de Rosario; ocho y diez de Córdoba y siete y nueve de Mendoza. Fuente: elaboración propia.

Los datos permiten hipotetizar que la corrupción es definida mediáticamente como un problema relacionado principalmente con el ámbito público, y específicamente con "la política". El hallazgo, que es consistente con estudios de caso en otros países (Palau y Davesa, 2013), da cuenta de su construcción como escándalo político.

La segunda variable a tener en cuenta, expresada en la tabla 1, es la dimensión geográfica. Si se analizan los niveles de cobertura mediática de cada provincia, en los noticieros del AMBA la corrupción acapara el 64,4\% de las noticias relacionadas con delitos, mientras que en Córdoba ocupa el 31,6\%, en Mendoza el 25,5 \% y en Rosario el 15,8\%. Los datos permiten establecer que los escándalos políticos asociados a corrupción se instalan fuertemente desde los medios del Área Metropolitana de Buenos Aires (AMBA) e irradian hacia los de las provincias, con un fuerte arraigo en casos relacionados con la política nacional. Es decir, la corrupción no se constituye como un problema público de la política local, sino principalmente que es asociada 
principalmente al poder central con sede en Buenos Aires. Y desde allí se instala como tema en los medios del interior, en buena medida a través de piezas informativas producidas desde los grandes medios de Buenos Aires.

Tabla 1. frecuencia de temas según ciudad del medio. Canales once y trece de Buenos Aires; tres y cinco de Rosario; ocho y diez de Córdoba y siete y nueve de Mendoza.

\begin{tabular}{lcccc}
\hline & Buenos Aires & Córdoba & Rosario & Mendoza \\
\hline Corrupción en el ámbito público & $64,4 \%$ & $31,6 \%$ & $15,8 \%$ & $25,5 \%$ \\
\hline Robo o hurto & $8,9 \%$ & $15,8 \%$ & $26,3 \%$ & $16,4 \%$ \\
\hline Amenaza & $4,4 \%$ & & $5,3 \%$ & $3,6 \%$ \\
\hline Violencia sexual & $4,4 \%$ & & $5,3 \%$ & $18,2 \%$ \\
\hline Otros & $4,4 \%$ & & $2,6 \%$ & $7,3 \%$ \\
\hline Asesinato & $2,2 \%$ & $5,3 \%$ & & $3,6 \%$ \\
\hline Narcotráfico & $2,2 \%$ & $5,3 \%$ & & $1,8 \%$ \\
\hline Femicidio & $2,2 \%$ & $21,1 \%$ & $2,6 \%$ & $1,8 \%$ \\
\hline Violencia infantil & $2,2 \%$ & $15,8 \%$ & $15,8 \%$ & $3,6 \%$ \\
\hline Violencia en las calles & $2,2 \%$ & & $2,6 \%$ & $10,9 \%$ \\
\hline Seguridad vial & $2,2 \%$ & & $5,3 \%$ & $3,6 \%$ \\
\hline Violencia de género & & & $2,6 \%$ & $1,8 \%$ \\
\hline Violencia institucional & & & $2,6 \%$ & \\
\hline Violencia en las cárceles & & & $13 \%$ & \\
\hline Corrupción en el ámbito privado & & & & \\
\hline
\end{tabular}

Fuente: elaboración propia.

Si se tiene en cuenta que las noticias sobre corrupción poseen lazos con otras asociadas a diferentes tipos de delito, como las de inseguridad, resulta interesante advertir la manera particular que en unas y otras se construyen arquetípicamente a las víctimas y victimarios. Mientras que las víctimas de la inseguridad se erigen principalmente como ciudadanos urbanos de clase media que son identificados explícitamente en las noticias a partir de un caso (Zunino y Focás, 2018), ocho de cada diez noticias sobre corrupción no identifican una víctima definida, mientas que las que sí lo hacen la cimentan genéricamente representada como "la gente". Tal denominación, que forma parte de un proceso más típico de construcción de identidades, remite a un "sujeto cambiante y desligado de las tradiciones políticas" (Vommaro, 2008, p. 25) a cuya 
identificación y reacción se apela. Así, la relación entre los "ciudadanos" o "vecinos" fundados desde un nosotros inclusivo que tiene como contradestinatario a la "clase política" se instituye como el par dicotómico que motoriza y estabiliza la controversia.

En tanto, el victimario arquetípico de la corrupción en el período que se estudia se instituye mediante la atribución de responsabilidad explícita a un actor central: el gobierno de la expresidenta Cristina Fernández de Kirchner (2007-2015). Tal como lo demuestra el gráfico 2, ella y sus exfuncionarios son constituidos como victimarios directos en el 47,3 \% de las noticias relevadas. Los organismos de derechos humanos con los que ese Gobierno estableció vínculos para la construcción de viviendas y otras obras de infraestructura social, como la organización Madres de Plaza de Mayo, son sindicados como victimarios en un 27,3 \% de las menciones. Los empresarios asociados con ese gobierno, señalados explícitamente como testaferros, como Lázaro Baez o Cristóbal López, aparecen en las noticias como victimarios el $18,2 \%$ de las veces. Finalmente, solo el 5,5\% de las noticias pone el foco en el poder judicial como partícipe de los escándalos de corrupción.

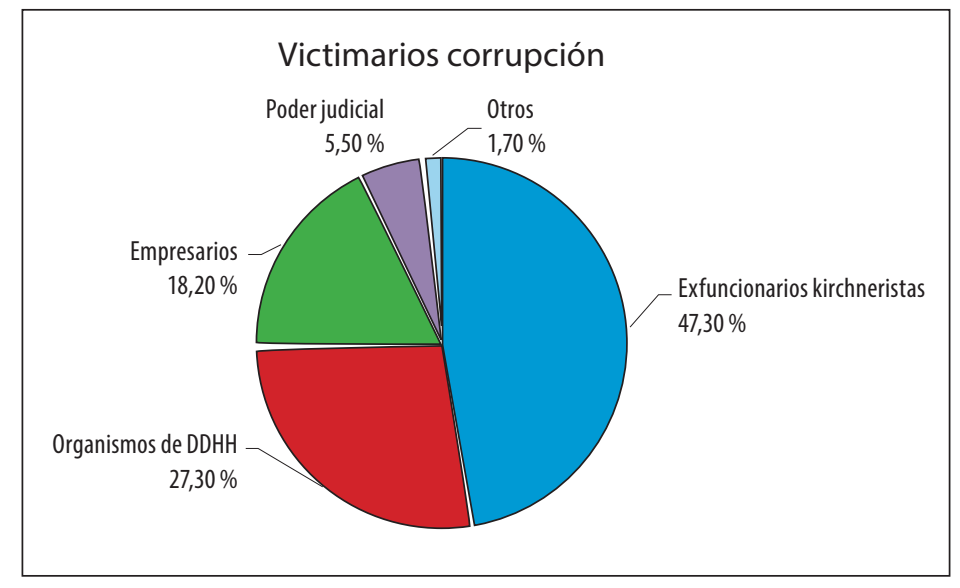

Gráfico 2. victimarios de casos de corrupción. Canales once y trece de Buenos Aires; tres y cinco de Rosario; ocho y diez de Córdoba y siete y nueve de Mendoza.

Fuente: elaboración propia.

Los datos expresan tendencias a contrastar sobre el modo de instauración de la "corrupción" como problema público durante el período de gobierno de Mauricio Macri (2015-2019), que en buena medida extienden sus rasgos centrales hasta el presente. La presentación del tema "corrupción" acompañado del adjetivo "K"3 da cuenta de una asociación que se estabilizó en las representaciones mediáticas dominantes y que opera cognitivamente como un argumento convincente (Ghanem, 1996; Kiousis,

\footnotetext{
${ }^{3}$ En referencia al apellido Kirchner
} 
2005). Este tipo de atributos, ampliamente abordados por la teoría de la Agenda Setting, constituyen una etiqueta que se asocia a un tema y, además de moldearlo, "tiene más probabilidades que otras de ser regularmente incluidas en los mensajes (...) y más probabilidades que otras de ser percibidas y recordadas por la audiencia" (McCombs, 2006, p. 179).

Por su parte, los puntos de vista presentes en una cobertura, analizados a partir del reconocimiento de las fuentes de información, son coherentes con la correlación de fuerzas percibida por los periodistas en una controversia política. Allí radica la visibilidad predominante que suelen adquirir las fuentes estatales y, más precisamente, las gubernamentales.

Sin embargo, tal condición no se dio en este caso, puesto que la visibilidad de las fuentes no oficiales duplica la presencia de las oficiales: $63,6 \%$ frente a $35,7 \%$. Tal como se analizó en trabajos previos sobre la cobertura del mismo tema en medios digitales (Koziner et ál., 2018, Zunino y Grilli fox, 2020), exfuncionarios, empresarios y personas ligadas con el gobierno de la expresidenta Cristina Fernández de Kirchner ocuparon un lugar sobresaliente como fuentes de información, pero sus puntos de vista fueron sistemáticamente desacreditados por la explícita evaluación moral negativa que los instituyó como culpables. En tanto, las fuentes oficiales recayeron principalmente en jueces, fiscales y otros miembros del poder judicial que se instalaron como actores centrales de los escándalos y de su proceso de construcción noticiosa. A ellos se sumaron, en menor medida, funcionarios del gobierno de Mauricio Macri, quienes también se consolidaron como actores denunciantes reiteradamente consultados por los medios de comunicación.

De este modo, la construcción de la corrupción como escándalo político en la Argentina de los últimos años cubrió cada uno de los aspectos constitutivos del concepto. En primer lugar, la publicidad a través de medios de comunicación a partir de una cobertura fuertemente dramatizada, personalizada y provista de elementos afectivos explícitos constituyó una controversia pública que tuvo como clivaje la infracción de una norma y su condena moral. Aunque los medios de comunicación actuaron no solo como engranajes del proceso de publicidad del escándalo, sino también como actores que encarnaron la figura del denunciante. Las investigaciones periodísticas, construidas a partir de filtraciones de datos por parte de fuentes oficiales, entre las que se destacan el poder judicial y los principales dirigentes de la alianza del gobierno de Mauricio Macri (2015-2019), se instauraron como el principal actor denunciante y articulador de escenarios plausibles en el que un victimario focalizado en un grupo de figuras políticas y empresariales cercanas a un sector político se instituyeron como amenaza y ofensa social. En tanto, la explicitación de los intereses afectados apeló a la identificación como víctima de un colectivo social indiferenciado o genérico 
al que se apeló, en tanto principal damnificado, para el ejercicio de una reacción emocional de indignación. Así, las condenas sociales tomaron distancia de las decisiones judiciales y se moldearon primero en los medios de comunicación mediante la creación de escenarios verosímiles o plausibles (Pereyra, 2014), cuya efectivad depende, en cada caso, del sistema de valores y encuadres que operan al nivel de los públicos.

\section{Las percepciones de las audiencias sobre la corrupción}

Para conocer las percepciones de las audiencias en relación con el tema, se expuso a los y las participantes de los grupos focales a una noticia de corrupción que estaba dentro del mismo corpus sobre el que se desarrolló el análisis de contenido. La noticia, que tenía a Lázaro Báez ${ }^{4}$ como protagonista y fue emitida por el noticiero Tele noche, trataba sobre un caso de sobreprecios y fraude al Estado. Y era presentada por los periodistas en el piso con música y movimientos de cámara, ya que se trataba de una nota exclusiva: "Lázaro Báez habla por primera vez desde la cárcel". El tono en que fue exhibida no pasó desapercibido por las audiencias quienes señalaron la construcción sensacionalista de la noticia:

Hombre: No deja de ser un show de...televisivo. Siempre va a existir un (Gobierno) malo y otro mejor y después...

Mujer: a veces hasta me resulta gracioso, como lo de la monjita con los bolsos, sí, yo creo que estuve como dos meses riéndome de eso porque me resultaba gracioso la situación. Un tipo tirando bolsos, la monjita que los agarraba, me resultó muy gracioso. (Grupo focal treinta-cincuenta años. NSE $\mathrm{Cl}$-C2. Residentes en GBA primer cordón, Norte, Sur y Oeste, Comunicación personal, 23 de agosto de 2016)

Mujer: ... Para lo que fue después porque en realidad, primero la música... urgente, último momento prácticamente parece que van a entregar a Cristina

Cristina: los mensajes encriptados

(Voces superpuestas)

Mujer: no tenía ninguna importancia lo que el tipo decía

Hombre: te hace compenetrarte y prestarle atención

Hombre: no contestó nada, en síntesis, no dijo nada.

Hombre: no, que capaz es lo que decía él, por ahí te hace enfocarte y mirarla y tratar de ver la noticia y estar pendiente de eso, por el título y esperando que pase algo. (Grupo focal veinticinco a cincuenta y cinco años. NSE medio. Residentes Córdoba capital, Comunicación personal, 23 de agosto de 2016)

\footnotetext{
${ }^{4}$ Lázaro Báez. Canal: Canal trece. Fecha de emisión 21 de agosto de 2016. Duración: seis minutos un segundo. Primer bloque.
} 
Una primera cuestión que surge de la relación entre el abordaje desde la recepción y el análisis de contenido es que, así como los medios presentan el tema en general en las secciones de política, los participantes también afirmaban que se trataba de una noticia "política".

¿Y esta noticia si la tuvieran que ubicar en una grilla o en un diario, en qué sección lo harían...?

Hombre: En política, política.

Mujer: Y sí, en política

Hombre: Sí, yo pensaba lo mismo, política porque son políticos los protagonistas...

Mujer1: Sí, no hay una sección estafa...

Mujer: iClaro!

Mujerl: Es una gran estafa, una estafa... (Grupo focal cuarenta-sesenta y cinco años. NSE C1 y C2. Residentes en CABA, todos los barrios, menos Sur, Comunicación personal, 23 de agosto de 2016)

¿Dónde ubicarían esta noticia? ¿En qué sección?

Hombre: política...empresarial porque están nombrando a empresarios de la obra publica

Hombre: ¿̇corrupción está dentro de política? O ces un rubro aparte?

Mujer: van juntos [coinciden todos]. (Grupo focal cuarenta-cincuenta y seis años. NSE D1 D2. Residentes en GBA segundo y tercer cordón, Comunicación personal, 23 de agosto de 2016)

Una particularidad que permitió dilucidar el estudio de recepción fue que, en comparación con las otras noticias que los participantes miraron (casos de inseguridad urbana y femicidios), en este tema era donde percibían mayor distancia en relación con su vida cotidiana. No se trataba de un desinterés total, sino que, al leer la noticia asociada a la cuestión política, el sentido se fundía en una mirada despectiva o cínica sobre el tema. "No hay nada que hacer", "siempre lo mismo", "se robaron todo y siguen robando", son algunos de los comentarios de las audiencias frente a la noticia. Como se dijo, el estudio en la instancia de recepción permitió confirmar la característica de la corrupción como tema "no experiencial", debido a su menor posibilidad de contrastación directa, lo que llevaría a una mayor incidencia de las construcciones mediáticas.

¿Qué opinan sobre esta noticia?

Mujer: a mí la política me pasa de largo 
¿Pero esta noticia? ¿Te aburrió?

Mujer: la omito

- ¿Harías zapping?

- Mujer: sí, sí, totalmente. Por ahí la empiezo a ver y digo "ah es política". No está en mis manos, estos son arreglos entre unos y otros; paso de largo. (Grupo focal: veinticinco a cincuenta y cinco años. NSE bajo. Residentes Rosario, Comunicación personal, 4 de septiembre de 2016)

Mujer: yo no la miraría, la leería. Sí, me interesaría la transcripción de lo que él dice en la conversación telefónica, por ahí la leería...Pero me molesta muchísimo el tema. (Grupo focal treinta-cincuenta años. NSE Cl -C2. Residentes en GBA primer cordón, Norte, Sur y Oeste, Comunicación personal, 4 de septiembre de 2016)

En síntesis, lo interesante es que, frente a un tema no experiencial, los participantes muestran un conocimiento sobre la construcción de la noticia, hablan espontáneamente de "sensacionalismo", enumeran características que consideran propias del medio audiovisual y expresan cierta familiaridad con los modos del trabajo periodístico y las lógicas de las empresas de medios, como veremos más adelante.

\section{Victimas- victimarios}

Otro de los cruces relevantes que observamos entre el análisis de producción y los grupos focales fue sobre las figuras de las víctimas y victimarios en este tipo de noticias. Como afirmamos, el análisis de contenido arroja que ocho de cada diez noticias de corrupción no identifican una víctima definida, mientas que las que sí lo hacen la construyen genéricamente representada como "la gente". Para Pitch (2009) la condición de víctima se transformó en premisa que legitima la acción pública, por lo que el empoderamiento aparece, paradójicamente, vinculado a la vulnerabilidad. Para vastos sectores detentar una voz legítima en la posmodernidad implica primero constituirse como víctimas, la apelación a la victimización padecida se convirtió en una de las modalidades más poderosas para hacerse oír. Luego de exponer la noticia sobre el caso de Lázaro Báez se solicitó a los participantes que identifiquen quiénes eran las víctimas y los victimarios. La víctima fue identificada como "el Estado", "todos nosotros", y "Lázaro Báez", según las distintas interpretaciones. En el momento de la reproducción de la noticia, emitida por Tele noche, Báez se encontraba en prisión preventiva. Las distintas interpretaciones en este caso se centraban sobre todo en que algunos atribuían un fuerte peso de la corrupción en el gobierno de Néstor y Cristina F. de Kirchner mientras que otros al ponerlo en duda, lo equiparaban con la corrupción en el gobierno de Mauricio Macri.

\section{¿Quién sería en esta noticia la víctima o las víctimas?}


Mujer: nosotros, la sociedad, porque se le robó, se comprobó y el dinero no aparece.

Varón: eso no es lo peor que el dinero no aparezca, peor que eso es que la persona que se está aclarando que robó todo sigue en libertad.

Mujer: y que no es él solo.

Varón: que no es él solo.

Varón: se está comprobando que ciertas personas fueron culpables no están presas.

Mujer: y que mientras sucede todo esto a nosotros nos siguen...

Mujer: mucha gente se enoja porque dicen si yo tuve una causa, qué sé yo, joven, hice alguna cosa, yo ya no puedo trabajar en el estado. Y esta gente se sigue postulando en la política...

Mujer: lo que provoca eso es el enojo en la gente. (Grupo focal: veinticinco a cincuenta y cinco años. NSE medio. Residentes en Mendoza, Comunicación personal, 14 de febrero de 2017

\section{¿Quién es la víctima en esta noticia?}

Mujerl: nosotros.

Mujer 2: nosotros, el pueblo. El pueblo.

Hombrel: nosotros como Estado, como nación. Como pasó todas las veces que ha pasado y el pueblo sigue pagando.

Mujer2: sí, el pueblo

Hombrel: cuando se vaya este el pueblo lo va a pagar y siempre, las noticias son siempre igual.

Mujerl: siempre, el pueblo. (Grupo focal veinticinco a cincuenta y cinco años. NSE medio. Residentes Rosario, Comunicación personal, 14 de febrero de 2017)

La adopción del punto de vista del ciudadano-victima por parte de los participantes acentuó posiciones polarizadas, tal como veremos en el próximo apartado. En tanto, los testimonios expresan que, en alguna medida, la apelación a la indignación ciudadana como rasgo estructurante de los escándalos de corrupción es verificable en el discurso de las audiencias.

\section{Polarización afectiva y saturación}

Si bien las noticias de corrupción promueven en principio poco interés para las audiencias, despiertan fuertes emociones y reacciones vinculadas con los posicionamientos 
previos de quienes las miran. En los grupos se entablaban discusiones sobre el caso de Lázaro Báez, en relación con la corrupción y las malversaciones de fondos del Estado, pero también sobre los gobiernos kirchneristas y su responsabilidad en el caso. En este sentido, se advirtió que la noticia promovía distintas emociones, recuperando la dimensión afectiva de los estudios de recepción.

Primera reacción, sentimientos, ¿qué les causa esta noticia?

Mujerl: impotencia.

Hombrel: risa.

Mujer2: cansancio.

Mujer3: hartazgo, exactamente.

Mujerl: impotencia de no poder con todo esto.

Hombre2: nos toman el pelo.

¿Quiénes nos toman el pelo?

Hombre2: como decía él hoy que uno se acuerda ponerle, el origen... Es como que él negaba que era empleado bancario, y si era empleado bancario...

Mujer4: sí, era empleado bancario.

Hombre3: era sabido, hay cosas que no se pueden ocultar.

Hombre2: Y se defiende diciendo ay, a mí ahora esto. Pero él manejó toda esa plata, si demostraron que tiene casi mil autos de colección. (Grupo focal cuarenta-sesenta y cinco años. NSE Cl y C2. Residentes en CABA, todos los barrios, menos Sur, Comunicación personal, 3 de abril de 2017).

¿Qué reacciones les genera esta noticia?

Mujerl: bronca

Hombre: y...una impotencia.

Bronca, impotencia. Una impotencia distinta a otras noticias que vimos o la misma...

Mujerl: a mí me parece que en las anteriores te generan hasta cierto dolor. Y esta impotencia tiene que ver con que hay aspectos y sectores, cosas a las que nunca vas a llegar y que esa corrupción siempre te influye a vos y a tu vida, por esas cosas del poder. Entonces una impotencia con enojo, con bronca.

Hombre: a mí me rebota casi lo que le pasa a ella porque digo, ¿̇cuándo va a pasar eso con un testaferro de Macri? ¿O de los (Roche) que no los conoce nadie y tienen tanta plata en este país y afuera? Por qué siempre la justicia... (Grupo focal veinticinco a cincuenta y cinco años. NSE medio. Residentes Mendoza, Comunicación personal, 3 de abril de 2017) 
Hipotetizamos entonces que el proceso de recepción de estas noticias incide en la generación de sentimientos contradictorios que péndula entre bronca, impotencia, angustia y hartazgo, lo que muchas veces lleva a dejar de mirar estas noticias. Algunos participantes manifestaban cierta saturación por estos temas, lo que los impulsaba a cambiar de canal.

Hablamos de los distintos delitos, hablamos de los delitos políticos, ¿miran las noticias sobre delitos políticos?

Mujerl: Yo saturada.

Estás muy saturada...

Hombrel: lo mismo porque o sea ya sabes que se chorea, se chorea y no pasa nada.

Mujer2: nunca veo un hecho como que sea concreto al final, o sea, es todo como que se amaga y no termina siendo.

Hombrel: y eso sí (VS)

Hombre2: y no te convencen las penas que le dan tampoco.

Hombre3: cuando les dan pena...

Mujer2: y siempre sigue más de lo mismo. (Grupo focal: veinticinco a cincuenta y cinco años. NSE bajo. Residentes Rosario, Comunicación personal, 3 de abril de 2017)

La saturación, entendemos, tiene lugar no solo por la repetición del tema en los medios, sino también producto de que el problema no termina de interpelarlos. De esta manera, para no exponerse a información sobre cuestiones delictivas, una salida posible era cambiar de canal, o directamente apagar la televisión. También en redes sociales, ignorar la información incidental que aparece sobre casos policiales. Esto, sin embargo, no significaba que los participantes no estén al tanto de esa información, ya que les llegaba por conversaciones, rumores, o por "escuchar de fondo", lo que pone de manifiesto que distanciarse es una constituye una decisión activa.

Otra característica de este tipo de textos noticiosos es la condena mediática anticipada. Es decir, la imputación de responsabilidades en casos sobre los que al momento de la noticia no suele haber condena judicial, lo que provoca una colisión entre el debate público y el principio de inocencia consagrado constitucionalmente. Así, la etiqueta "corrupción K" se constituyó en sí misma en un tipo de delito que, atribuido mediáticamente a un sector político y empresarial en particular, remite a una gran cantidad de causas judiciales que en su mayoría están en etapa de instrucción y que, por lo tanto, no tienen condena firme. 
También la orientación editorial del medio que presenta la noticia, en este caso, el noticiero Tele noche de Canal trece de Buenos Aires, funciona como una mediación significativa para los participantes.

Hombre: lo que pasa es que en este informe lo que hay que entender es que es obvia la animosidad. Hay una pelea vieja entre el grupo, Canal 13, del grupo Clarín, y el gobierno que pasó. Entonces, no puede ser presentado de otra manera. Porque además el mayor caudal de denuncias del gobierno anterior, en cuanto a corrupción, vino del mismo grupo. Y no estoy juzgando. (Grupo focal treinta-cincuenta años. NSE C1 -C2. Residentes en GBA primer cordón, Norte, Sur y Oeste, Comunicación personal, 10 de julio de 2017)

Hombre: cada canal lo va a mostrar de acuerdo a la tendencia, bajan la línea. Y va a estar Morales de un lado, y Lanata del otro. Siempre. (Grupo focal cuarenta-sesenta y cinco años. NSE C1 y C2. Residentes en CABA, todos los barrios, menos Sur, Comunicación personal, 10 de julio de 2017)

La noticia de corrupción polariza, pero se hallaron también miradas divergentes sobre el tema. A modo de ejemplo, en relación con la corrupción, los públicos pueden considerar que la noticia presentada está "inflada", que no se sabe nada, que el gobierno de Macri es corrupto, pero al mismo tiempo detestar a Cristina Fernández de Kirchner. O decir que roban en ambos gobiernos. Alguien puede exculpar al gobierno kirchnerista, pero igualmente estar sumamente enojado y sentirse víctima en tanto miembros de la sociedad por los robos de la corrupción.

\section{Tensión local- nacional}

Un último interrogante apunta a indagar sobre la tensión entre lo nacional y lo local. Los datos del análisis de contenido permiten establecer que la corrupción no se constituye como un problema público de la política local, sino principalmente asociada al poder central con sede en Buenos Aires, que luego se instala como tema en los medios de las provincias. En los grupos focales desarrollados en el interior (Mendoza, Córdoba y Rosario) se dio una particularidad en el proceso de recepción de la noticia sobre el caso Lázaro Báez. Si bien los y las participantes reaccionaron igual que los de los grupos del AMBA en relación con los sentimientos (bronca, hartazgo, tristeza), los públicos de las provincias tendieron a realizar una lectura situada del tema. Por lo tanto, el contexto local incidió en las interpretaciones, ya que los participantes asociaron el caso Báez con otros casos de corrupción y/o de impunidad vinculados al poder provincial, poco habituales en la superficie mediática nacional. También denunciaron que, según su percepción, los canales locales replican los casos nacionales y que de la corrupción local no hablan, ya que los noticieros "tapan esos temas" por acuerdos con los gobiernos en el poder. 
¿Al resto les genera algo?

Hombrel: ...es lo mismo con el gobernador que tuvimos acá, el radical...

Hombre2: Angeloz se robó los bancos, se robó toda la plata... (Grupo focal veinticinco a cincuenta y cinco años. NSE bajo. Residentes Córdoba, Comunicación personal, 3 de abril de 2017)

¿Con esta noticia qué les pasa? ¿Tiene algún vínculo con su vida cotidiana o les parece que es algo...?

Hombrel: [me recuerda] a los revuelos que hay en el barrio Universidad, estuvo 10 años para que se (devolviera) y encima casi no sé...

Mujerl: no, no los han devuelto.

Mujer2: no los han devuelto y no los van a devolver.

Mujer3: bueno, ahí está la diferencia Canal 9 y Canal 7. Esa noticia no se vio en canal 7.

Mujer2: los medios de comunicación se vinculan directamente con la política.

Mujer3: sí, totalmente.

(voces superpuestas)

Hombre: y cuando voy a la montaña hay lugares donde yo hace 15 años atrás podía ir tranquilamente porque era una reserva. Y ahora dice cuidado (...) armado ${ }^{5}$. (Grupo focal veinticinco a cincuenta y cinco años. NSE bajo. Residentes Mendoza, Comunicación personal, 3 de abril de 2017)

Mujer: a mí me pasa, por ejemplo, ¿̇recuerdan el chico que mató a la chica del ... General Paz, el del auto, el del hijo...

(Varios dicen, ah, sí)

Mujer: como por tanta reacción en los medios, al tipo lo meten preso. Yo tengo conocidos que trabajan ahí en el coso de menores, a los meses salió. Eso no lo pasaron en ningún medio, porque sabían que era hijo de... El tema es que ese chico cuando lo sacaron no salió en ningún lado, de hecho puedo asegurar que ninguno de ustedes sabía que ese chico no estaba preso. Y eso no lo pasan.

¿Cómo fue el caso?

${ }^{5}$ Los participantes se refieren a Daniel Vila, accionista mayoritario del Grupo América, segundo multimedio del país. El empresario enfrentó causas judiciales y fallos por apropiación de terrenos correspondientes a la Universidad Nacional de Cuyo. 
Mujer: una mini Cooper, que acá...como que no es muy común. Entonces fue como iwow!. El rico alcoholizado, pisó y la mató a la chica.

Hombre: después murió la familia, la madre. Al poco tiempo.

Mujer: ... uno cree que se hizo justicia y no se hizo. (Grupo focal veinticinco a cincuenta y cinco años. NSE medio. Residentes Córdoba capital, Comunicación personal, 3 de abril de 2017)

Los testimonios muestran un hallazgo que abre a nuevas lecturas, ya que los entrevistados realizaron un proceso de recepción poniendo de relevancia un tipo de lectura negociada (Hall, 1980; Morley, 1996), en la que comprenden la codificación del mensaje, pero realizan lecturas situadas y acordes a sus contextos.

\section{Discusión y conclusiones}

En este artículo se ensayaron relaciones entre el análisis de los contenidos mediáticos relacionados con el tema corrupción y su proceso de recepción. Esta mirada permitió observar algunas simetrías entre ambos procesos, así como divergencias y polémicas entre lo que las noticias emiten y las interpretaciones de las audiencias.

Algunos indicios nos permiten inferir al menos tres cuestiones centrales. En primer lugar, la existencia de cierta consonancia entre el marco en el que los medios presentan la noticia y el lugar que ocupa el tema desde la mirada de las audiencias. Desde ambos lados, la noticia de corrupción se enmarca como política.

Sin embargo, si bien los delitos de corrupción tuvieron un lugar trascendental en las agendas mediáticas durante el gobierno de Cambiemos, las audiencias manifestaron poco interés por este tipo de noticias. Es posible hipotetizar que esta distancia frente al tema (en relación con otros delitos) puede estar influida por los marcos que operan al nivel de los públicos, ya que la política ha sufrido un proceso de denostación muy fuerte en los últimos años. Encuadre que aparece de manera evidente y mayoritaria en los testimonios recogidos.

Una segunda cuestión que surge del análisis se vincula con que la construcción noticiosa en términos partisanos podría incidir en la forma en que se interpreta la noticia. No solo la presentación sensacionalista y los comentarios de los periodistas en el piso impactan en las audiencias. Los roles construidos en los discursos mediáticos interpelan a los públicos como víctimas. Sin embargo, el tema "corrupción" fue el que más polarización afectiva generó en los grupos en relación con sus ideologías oficialistas u opositoras. En tanto, hubo participantes que marcaron distancia entre esos dos polos. Fueron aquellos que en general proclamaron una mirada desilusionada sobre la política. En consonancia, el noticiero que emitía la noticia generó controversias a partir de la identificación por parte del público de su línea editorial opositora, lo que 
evidenció un conocimiento de las audiencias de las estructuras de propiedad de los grupos mediáticos y sus orientaciones político-ideológicas más habituales.

Por último, la constatación de una cobertura porteñocéntrica de la corrupción, si bien tuvo cierta efectividad en la publicidad de los temas al instalarlos de manera relevante en sus agendas, no impidió una lectura situada, basada en la interpretación de los sucesos desde las experiencias con la política local.

Los resultados del trabajo de investigación descriptos hasta aquí constituyen avances que permiten dilucidar algunas de las interacciones y tensiones que tienen lugar entre los contenidos de las noticias y su recepción, tomando como referencia un caso controvertido de corrupción. La indagación sobre otros temas y dimensiones de análisis contribuirán a completar un complejo mapa de interacciones complejas. Allí, tanto las miradas deterministas como las negadoras de importancia de las representaciones mediáticas resultan insuficientes. Nuevos estudios de caso a partir de evidencia empírica y triangulación metodológica posibilitarán avanzar en la construcción de nuevas hipótesis y siempre provisorias sobre una relación tan espinosa como apasionante.

\section{Referencias}

Abeles, M. y Borzel, M. (2004). Metas de inflación: implicancias para el desarrollo. Centro de economía y finanzas para el desarrollo de la argentina.

Arboleda, L. (2008). El grupo de discusión como aproximación metodológica en investigaciones cualitativas. Revista Facultad Nacional de Salud Pública, 26(1), 69-77.

Aruguete, N., Anselmino, N. R., Zunino, E. A., Koziner, N. S. y Fabbro, G. (2018). Matriz para el estudio de noticias televisivas sobre delito, violencia e inseguridad: una articulación teóricometodológica. Austral Comunicación, 7(2), 229-250. http://www.austral.edu.ar/ojs/index.php/ australcomunicacion/article/view/242/pdf_1

Aruguete, N. y Calvo, E. (2020). Coronavirus en Argentina. Polarización partidaria, encuadres mediáticos y temor al riesgo. Revista Saap, 14(2), 281-310. https://doi.org/10.46468/ rsaap. 14.2.a2

Aruguete, N. y Calvo, E. (2018). Time to \#protest: Selective exposure, cascading activation, and framing in social media. Journal of Communication, 68(3), 480-502. https://doi.org/10.1093/joc/ jqy007

Bagdikian, B. H. (1985). The U. S. Media Supermarket or Assembly Line? Journal of Communication, 3(35), 97-109. https://doi.org/https://doi.org/10.1111/j.1460-2466.1985.tb02451.x

Bennett, W. L. (1991). News: The Politics of Illusion, Ninth Edition. Longman.

Bennett, W. y Livingston, S. (2018). The Disinformation Order: Disruptive Communication and the Decline of Democratic Institutions. European Journal of Communication, 33(2), 122-139. https:// doi.org/10.1177/0267323118760317 
Bennett, W. y Pfetsch, B. (2018). Rethinking Political Communication in a Time of Disrupted Public Spheres. Journal of Communication, 68(2), 243-253. https://doi.org/10.1093/joc/jqx017

Berger, P. y Luckmann, T. (1968). La construcción social de la realidad. Amorrortu.

Bloor, M., Frankland, J., Thomas, M. y Stewart, K. (2000). Focus Groups in Social Research (Introducing Qualitative Methods Series). SAGE.

Colle, R. (201 1). El análisis de contenido de las comunicaciones. Sociedad Latina de Comunicación Social.

Entman, R. M. (1993). Framing: Toward Clarification of a Fracture Paradigm. Journal of Communication, 43(4), 51-58.

Fernández Pedemonte, D. (2010). Conmoción pública. Los casos mediáticos y sus públicos. La Crujía.

Gallart, M. (2002). Veinte años de educación y trabajo: la investigación de la formación y la formación de una investigadora. Revista mexicana de investigación educativa, 13(37), 649-655. https://biblat.unam.mx/es/revista/revista-mexicana-de-investigacion-educativa/articulo/ gallart-maria-antonia-veinte-anis-de-educacion-y-trabajo-la-investigacion-de-la-formaciony-la-formacion-de-una-investigadora-montevideo-cinteforoit-2002

García Santamaría, J. V. (2010). Crisis del periodismo de fuentes. Las prácticas del periodismo en España en el accidente de Spanair. Revista Latina de Comunicación Social, 65, 516-537. https:// doi.org/10.4185/RLCS-65-2010-916-516-537

Ghanem, S. (1996). Media coverage of crime and public opinion: an exploration of the second level of agenda setting. University of Texas.

Hall, S. (1980) Encoding and Decoding in the Television Discourse. Paper for the Council of Europe Colloquyon Training in the Critical Reading of Televisual Language. Centre for Contemporary Cultural Studies, University of Birmingham, Stencilled Occasional Paper, (7).

Igartua, J. y Humanes, M. (2004). Métodos cuantitativos de investigación en comunicación. Bosch.

Iyengar, S. (2001). Framing Responsibility for Political Issues. Hispanic Journal of Behavioral Sciences, 9(2), 183-205. https://doi.org/10.1177/07399863870092005

Kessler, G. (2009). El sentimiento de inseguridad. Sociología del temor al delito. Siglo XXI Editores.

Kessler, G. (2014). Controversias sobre la desigualdad. Argentina, 2003-2013. Fondo de Cultura Económica.

Kiousis, S. (2005). Compelling Arguments and Attitude Strength: Exploring the Impact of SecondLevel Agenda Setting on Public Opinion of Presidential Candidate Images. The Harvard International Journal of Press/Politics, 10(2), 3-27. https://doi.org/10.1177/1081 180X05276095

Kitsuse, J. y Spector, M. (2001). Constructing Social Problems. Routledge. https://doi.org/https://doi. org/10.4324/9781315080512

Koziner, N., Aruguete, N. y Zunino, E. (2018). Las fuentes de la corrupción. Voces en el Fenix, 74, 77-81. https://www.vocesenelfenix.com/sites/default/files/pdf/10_48.pdf

Krippendorff, K. (1990). Metodología de análisis de contenido. Teoría y práctica. Paidós. 
Martini, S. (2017). Últimas noticias. Construyendo la actualidad en el siglo XXI. La noticia hoy. Tensiones entre la política, el mercado y la tecnología. Imago Mundi.

McCombs, M. (2006). Estableciendo la agenda. El impacto de los medios en la opinión pública y el conocimiento. Paidós Ibérica.

McCombs, M. y Valenzuela, S. (2014). Agenda-Setting Theory: The Frontier Research Questions. Encyclopedia of communication theory, 1, 32-33 https://doi.org/10.4135/9781412959384.n12

Morley, D. (1996). Televisión, audiencias y estudios culturales. Amorrortu Editores.

Neuendorf, K. (2002). The content analysis guidebook. Sage.

Palau, A. y Davesa, F. (2013). El impacto de la cobertura mediática de la corrupción en la opinión pública española. Revista Española de Investigaciones Sociologicas, 144(1), 97-126. https://doi. org/10.5477/cis/reis. 144.97

Pereyra, S. (2009). La corrupción como problema público en la Argentina de los años '90: un análisis de las actividades de denuncia. 2009 Congress of the Latin American Studies Association.

Pereyra, S. (2012). La política de los escándalos de corrupción desde los años 90. Desarrollo Económico, 52(206), 255-284.

Pereyra, S. (2014). La corrupción como crítica moral de la política. El vocabulario de la protesta social durante la década de los 90. Papeles de Trabajo, 8(13), 78-101.

Pitch, T. (2009). La sociedad de la prevención. Ad hoc.

Reese, S. D. (2001). Framing Public Life: A Bridging Model for Media Research. Framing Public Life: A Bridging Model for Media Research. Lawrence Erlbaum.

Steele, J. (1997). Don`t Ask, Don`t Tell, Don`t Explain: Unofficial Sources and Television Coverage of the Dispute Gays in the Military. Political Communication, 14, 83-96. https://doi.org/https:// doi.org/10.1080/105846097199551

Thompson, J. (2012). El escándalo político. Paidós.

Vommaro, G. (2008). Lo que quiere la gente. Los sondeos de opinión y el espacio de la comunicación política en Argentina. Prometeo; Universidad Nacional de General Sarmiento.

Waisbord, S. (2020). ¿Es válido atribuir la polarización política a la comunicación digital? Sobre burbujas, plataformas y polarización afectiva. SAAP, 14(2), 249-279.

Zucker, H. (1978). The Variable Nature of News Media Influence. Communication Yearbook 2. Transaction Publishers.

Zunino, E. y Grilli Fox, A. (2020). Medios digitales en la Argentina: posibilidades y límites en tensión. Estudios sobre el Mensaje Periodístico, 26(1), 401 413. https://doi.org/10.5209/esmp.67320 https://doi.org/10.5209/esmp.67320

Zunino, E. y Focás, B. (2018). The Media's Coverage of "Insecurity" in Argentina: Victims, Victimisers and Lawsuits. Communication \& Society, 31, 189-209. https://dadun.unav.edu/ bitstream/10171/55790/1/zunino.pdf 